\title{
Efficient inverse position transformation for TR 4000S robot manipulator
}

\author{
KESHENG WANG $\dagger$
}

Keywords: inverse kinematics, robot control, robot manipulator, robotics.

\begin{abstract}
An efficient method is developed for computing the inverse kinematic position solution with a closed form for the TR 4000 S spray painting robot manipulator with five degrees of freedom and non-spherical wrist construction. The inverse kinematic problem is defined as the transformation from Cartesian space to the joint space. The solution is based on the geometrical separation of the arm and wrist of a robot manipulator and shows that it is very systematic, efficient and easily derived.
\end{abstract}

\section{Introduction}

A robot manipulator consists of a number of links connected together by joints. In order to position and orient the end effector of the robot manipulator arbitrarily in space, six degrees of freedom are necessary: three degrees of freedom for the position and three degrees of freedom for the orientation. Each robot manipulator joint can provide one degree of freedom, and thus a robot manipulator must have a minimum of six joints if it is to provide six orthogonal degrees of freedom in special position and orientation.

However, many tasks involve operations requiring fewer degrees of freedom, which can be realized by robot manipulators supplied with fewer than six joints. Such tasks appear, for instance, in the manipulation of axisymmetric objects such as turned workpieces, cylindrical tools and spray painting. These operations are rather frequent in industry, that is why five-degree-of-freedom robot manipulators are so common.

The computational efficiency of inverse kinematics is very important in robot manipulator research. In many path control schemes and motion planning, for instance, it is necessary to calculate the inverse kinematics of a robot manipulator at fairly high rates.

Inverse kinematics with a closed form solution for robot manipulators is attracting more and more attention from robot researchers, because it is much faster and more stable than numerical solutions in computation. The inverse kinematics with a closed form has been investigated for a six-degree-of-freedom robot manipulator and effective algorithms have also been developed (Featherstone (1983), Hollerbach and Sahar (1983), Low and Dubey (1986), Paul (1981)). However these algorithms can only be suitable for a special robot manipulator with a spherical wrist where the wrist axes intersect at one point.

Received 9 February 1989.

$\uparrow$ The Division of Production Engineering, Department of Mechanical Engineering, NTH, 7034 Trondheim, Norway. 
A more general algorithm for treating a six-degree-of-freedom robot manipulator with a non-spherical wrist has been developed by Wang and Lien (Wang (1988), Wang and Lien $(1987 \mathrm{a}, \mathrm{b})$ ). This approach is based on separating such a six-degreeof-freedom robot manipulator into two parts: the arm with the first three joints for the major positioning, and the wrist with the last three joints for the major orientation. The basic construction model for robot manipulators which are commercially available is considered, and these have five types of arm and two types of wrist. The approach for computing the inverse kinematics of robot manipulators is very systematic, efficient and easily derived.

After the six-degree-of-freedom robot manipulator with a non-spherical wrist has been dealt with to provide a set of inverse kinematics solutions with a closed form, attention is turned to the five-degree-of-freedom robot manipulators with a non-spherical wrist. According to author's knowledge, no such solutions with a closed form for five-degree-of-freedom robot manipulator have been published. This paper will describe how the approach for six-degree-of-freedom robot manipulators can be extended to obtain inverse kinematic solutions with a closed form for fivedegree-of-freedom robot manipulators. The TR $4000 \mathrm{~S}$ spray painting robot manipulator serves as an example.

\section{General principles}

At first, we will briefly review the approach which has been developed to compute the inverse kinematic solution with closed form for the six-degree-offreedom robot manipulators with a non-spherical wrist commonly used in industry (Wang and Lien (1987a)).

Though it initially appears to be difficult to find the inverse kinematic equations of a robot manipulator, when the robot manipulator is separated into two parts, the task becomes relatively simple and direct. The position and orientation of the end effector of the robot manipulator ${ }_{6}^{0} \mathrm{~T}$ is the product of the matrices (Pieper (1969)).

$$
{ }_{6}^{0} \mathrm{~T}=A_{1} A_{2} A_{3} A_{4} A_{5} A_{6}
$$

By the associative law, the product of the matrices can be regrouped into two subsets which represent the arm and wrist respectively.

$$
{ }_{6}^{0} \mathrm{~T}=\left(A_{1} A_{2} A_{3}\right)\left(A_{4} A_{5} A_{6}\right)
$$

where

$$
A_{1} A_{2} A_{3}={ }_{3}^{0} \mathrm{~T}={ }_{a}^{0} \mathrm{~T}
$$

and

$$
A_{4} A_{5} A_{6}={ }_{6}^{3} \mathrm{~T}={ }_{w}^{a} \mathrm{~T}
$$

The superscripts designate the reference frame; a represents the tip of the arm; and $w$ represents the tip of wrist, i.e., the centre of the end effector of a robot manipulator.

The ${ }_{6}^{0} \mathrm{~T}$ given for the end effector can be written as a $4 \times 4$ homogeneous matrix composed of an orientation submatrix $R$ and a position vector $\boldsymbol{P}$ (Paul (1981)). 


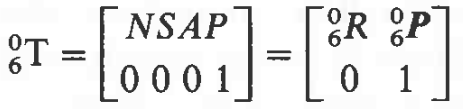

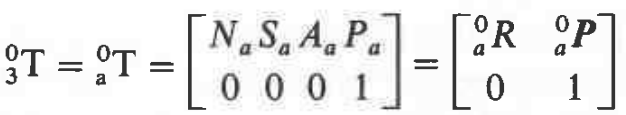

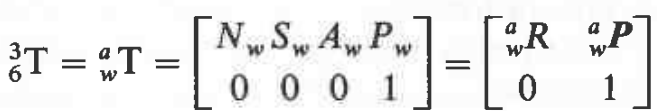

We can get the vector ${ }_{a}^{0} P$ directly using a vector analysis method. The detail will be considered in the next section.

$$
{ }_{a}^{0} P={ }_{6}^{0} P-{ }_{w}^{0} P
$$

From Eqn. (1),

$$
A_{1} A_{2} A_{3}=\left[\begin{array}{cc}
{ }_{3}^{0} R\left(\theta_{1} \theta_{2} \theta_{3}\right) & { }_{3}^{0} \boldsymbol{P}\left(\theta_{1} \theta_{2} \theta_{3}\right) \\
0 & 1
\end{array}\right]
$$

We can get $\theta_{1}, \theta_{2}, \theta_{3}$, the first three joint variables, from the solution of the following equation:

$$
{ }_{3}^{0} \boldsymbol{P}\left(\theta_{1} \theta_{2} \theta_{3}\right)={ }_{a}^{0} \boldsymbol{P}
$$

The orientation of the end effector of the robot manipulator can be considered as the product of the orientation of the arm and the orientation of the wrist:

$$
{ }_{6}^{0} R={ }_{3}^{0} R{ }_{6}^{3} R={ }_{a}^{0} R{ }_{w}^{a} R
$$

From Eqns. (2) and (3) we can obtain

$$
{ }_{6}^{3} R=\left({ }_{3}^{0} R\right)^{-1}{ }_{6}^{0} R={ }_{w}^{a} R
$$

where

$$
\begin{aligned}
{ }_{6}^{0} R & =[N S A] \\
\left({ }_{3}^{0} R\right) & =\text { rotation part of matrices }\left(A_{1} A_{2} A_{3}\right) \\
\left({ }_{6}^{3} R\right) & =\text { rotation part of matrices }\left(A_{4} A_{5} A_{6}\right)
\end{aligned}
$$

We can get the last three joint variables $\theta_{4}, \theta_{5}, \theta_{6}$ by solving the Eqn. (4).

Now we will consider five-degree-of-freedom robot manipulators which lose one degree of freedom in the wrist configuration, so that it cannot reach the any arbitrary place in space. A five-degree-of-freedom robot manipulator can simply be considered as a six-degree-of-freedom robot manipulator in which one joint variable is fixed as zero or some suitable value. The lost degree of freedom may either be in the arm or wrist part of the robot manipulator. In either case, the solution of inverse kinematics can, in principle, be obtained by the application of the existing algorithm for a six-degree-of-freedom robot manipulator. The only necessary additional step is to determine which joint can represent the lost degree of freedom and this is not so difficult.

In most practical cases, such as parts handling, assembling and spray painting, one degree of freedom in orientation may be unnecessary, so one joint of wrist 
construction can be omitted. Then it will be simple to treat five-degree-of-freedom robot manipulators, since we only need to assign zero or some other suitable value to the joint variable of wrist. For example, if the degree of freedom of rotation around the approach direction is not necessary, the $\theta_{6}$ can be assigned a value of $90^{\circ}$, the approach for a six-degree-of-freedom robot manipulator can then easily be extended to solve five-degree-of-freedom robot manipulators. In the next section, we will use TR 4000S as a example to demonstrate how to extend the approach from six-degree-of-freedom to five-degree-of-freedom robot manipulators.

\section{Coordinate frames for TR $4000 S$ robot manipulator}

The configuration of TR 4000 S spray painting robot manipulator is shown in Fig. 1. The axes of its joint coordinate frames are assigned according to the rules established by Denavit-Hartenberg (Denavit and Hartenberg (1955)). Considering Fig. 2, the link parameter is given in Table 1.

Based on the generalized D-H transformations the following homogeneous transformation matrix derived for the $i$ th joint is given as:

$$
A_{i}=\left[\begin{array}{cccc}
c \theta_{i} & -s \theta_{i} c \alpha_{i} & s \theta_{i} s \alpha_{i} & a_{i} c \theta_{i} \\
s \theta_{i} & c \theta_{i} c \alpha_{i} & -c \theta_{i} s \alpha_{i} & a_{i} s \theta_{i} \\
0 & s \alpha_{i} & c \alpha_{i} & d_{i} \\
0 & 0 & 0 & 1
\end{array}\right] .
$$

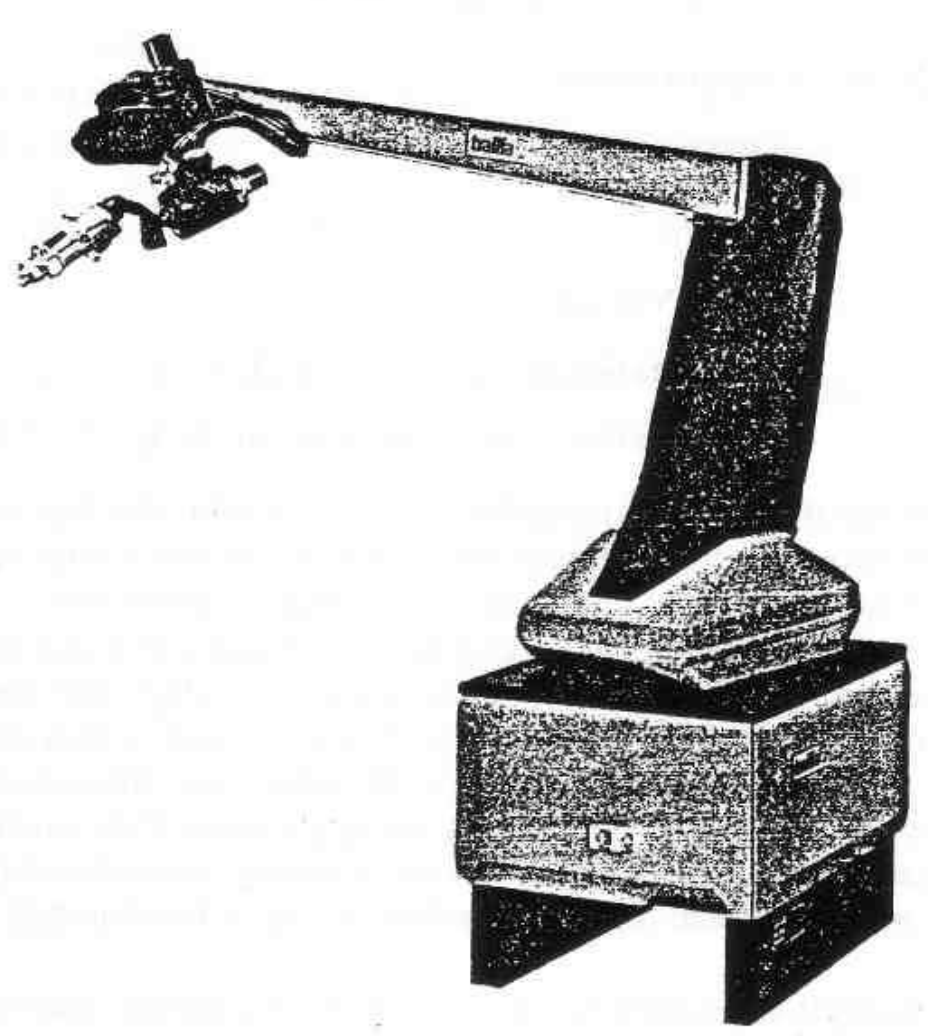

Figure 1. TR 4000S spray painting robot manipulator. 


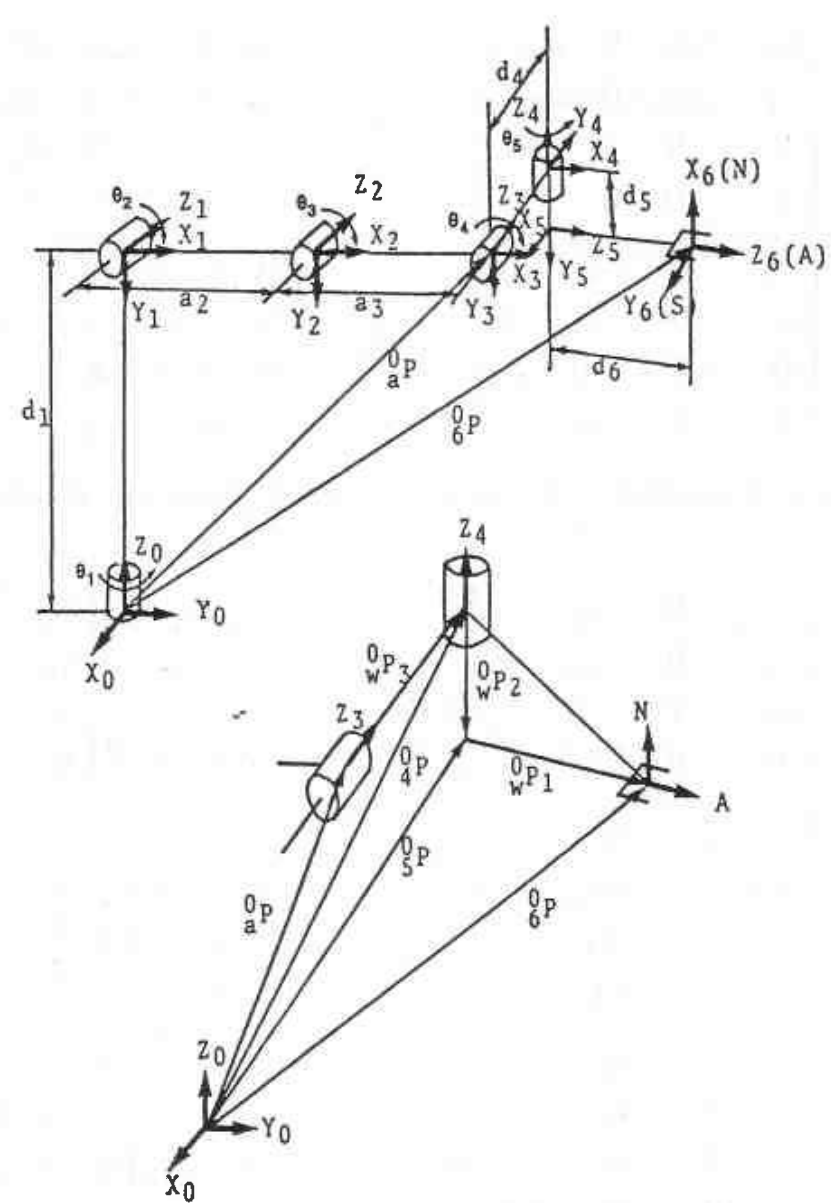

Figure 2. The coordinate frame for TR $4000 \mathrm{~S}$ robot manipulator.

All the transformation matrices for the TR $4000 \mathrm{~S}$ robot manipulator are given as follows:

$$
A_{1}=\left[\begin{array}{rrrr}
c_{1} & 0 & -s_{1} & 0 \\
s_{1} & 0 & c_{1} & 0 \\
0 & -1 & 0 & d_{1} \\
0 & 0 & 0 & 1
\end{array}\right] \quad A_{2}=\left[\begin{array}{rrrc}
c_{2} & -s_{2} & 0 & a_{2} c_{2} \\
s_{2} & c_{2} & 0 & a_{2} s_{2} \\
0 & 0 & 1 & 0 \\
0 & 0 & 0 & 1
\end{array}\right]
$$

\begin{tabular}{ccccc}
\hline Joint & $\theta_{\mathrm{i}}$ & \multicolumn{1}{c}{$\alpha_{\mathrm{i}}$} & $a_{\mathrm{i}}$ & $d_{\mathrm{i}}$ \\
\hline 1 & $\theta_{1}$ & $-90^{\circ}$ & 0 & $d_{1}$ \\
2 & $\theta_{2}$ & 0 & $a_{2}$ & 0 \\
3 & $\theta_{3}$ & 0 & $a_{3}$ & 0 \\
4 & $\theta_{4}$ & $90^{\circ}$ & 0 & $d_{4}$ \\
5 & $\theta_{5}$ & $-90^{\circ}$ & 0 & $d_{5}$ \\
6 & $90^{\circ}$ & 0 & 0 & $d_{6}$ \\
\hline
\end{tabular}

Table 1. Link parameters for TR 4000 S robot manipulator. 


$$
\begin{aligned}
& A_{3}=\left[\begin{array}{cccc}
c_{3} & -s_{3} & 0 & a_{3} c_{3} \\
s_{3} & c_{3} & 0 & a_{3} s_{3} \\
0 & 0 & 1 & 0 \\
0 & 0 & 0 & 1
\end{array}\right] \quad A_{4}=\left[\begin{array}{cccc}
c_{4} & 0 & s_{4} & 0 \\
s_{4} & 0 & -c_{4} & 0 \\
0 & 1 & 0 & d_{4} \\
0 & 0 & 0 & 1
\end{array}\right] \\
& A_{5}=\left[\begin{array}{rrrr}
c_{5} & 0 & -s_{5} & 0 \\
s_{5} & 0 & c_{5} & 0 \\
0 & -1 & 0 & d_{5} \\
0 & 0 & 0 & 1
\end{array}\right] \quad A_{6}=\left[\begin{array}{cccc}
0 & 1 & 0 & 0 \\
1 & 0 & 0 & 0 \\
0 & 0 & 1 & d_{6} \\
0 & 0 & 0 & 1
\end{array}\right]
\end{aligned}
$$

The transformation matrices for the arm and wrist parts are respectively given as follows:

$$
\begin{aligned}
{ }_{3}^{0} \mathrm{~T}=A_{1} A_{2} A_{3} & =\left[\begin{array}{cccc}
c_{1} & 0 & -s_{1} & 0 \\
s_{1} & 0 & c_{1} & 0 \\
0 & -1 & 0 & d_{1} \\
0 & 0 & 0 & 1
\end{array}\right]\left[\begin{array}{cccc}
c_{2} & -s_{2} & 0 & a_{2} c_{2} \\
s_{2} & c_{2} & 0 & a_{2} s_{2} \\
0 & 0 & 1 & 0 \\
0 & 0 & 0 & 1
\end{array}\right]\left[\begin{array}{cccc}
c_{3} & -s_{3} & 0 & a_{3} c_{3} \\
s_{3} & c_{3} & 0 & a_{3} s_{3} \\
0 & 0 & 1 & 0 \\
0 & 0 & 0 & 1
\end{array}\right] \\
& =\left[\begin{array}{cccc}
c_{1} c_{23} & -c_{1} s_{23} & -s_{1} & c_{1}\left(a_{3} c_{23}+a_{2} c_{2}\right) \\
s_{1} c_{23} & -s_{1} s_{23} & c_{1} & s_{1}\left(a_{3} c_{23}+a_{2} c_{2}\right) \\
-s_{23} & -c_{23} & 0 & -a_{3} s_{23}-a_{2} s_{2}+d_{1} \\
0 & 0 & 0 & 1
\end{array}\right] \\
{ }_{6}^{3} \mathrm{~T}=A_{4} A_{5} A_{6} & =\left[\begin{array}{cccc}
c_{4} & 0 & s_{4} & 0 \\
s_{4} & 0 & -c_{4} & 0 \\
0 & 1 & 0 & d_{4} \\
0 & 0 & 0 & 1
\end{array}\right]\left[\begin{array}{cccc}
c_{5} & 0 & -s_{5} & 0 \\
s_{5} & 0 & c_{5} & 0 \\
0 & -1 & 0 & d_{5} \\
0 & 0 & 0 & 1
\end{array}\right]\left[\begin{array}{cccc}
0 & 1 & 0 & 0 \\
1 & 0 & 0 & 0 \\
0 & 0 & 1 & d_{6} \\
0 & 0 & 0 & 1
\end{array}\right] \\
& =\left[\begin{array}{cccc}
-s_{4} & c_{4} c_{5} & -c_{4} s_{5} & -d_{6} c_{4} s_{5}+d_{5} s_{4} \\
c_{4} & s_{4} c_{5} & -s_{4} s_{5} & -d_{6} s_{4} s_{5}-d_{5} c_{5} \\
0 & s_{5} & c_{5} & d_{6} c_{5}+d_{4} \\
0 & 0 & 0 & 1
\end{array}\right]
\end{aligned}
$$

where

$$
c_{\mathrm{i}} \equiv \cos \theta_{\mathrm{i}} ; \quad s_{\mathrm{i}} \equiv \sin \theta_{\mathrm{i}} ; \quad c_{\mathrm{ij}} \equiv \cos \left(\theta_{\mathrm{i}}+\theta_{\mathrm{j}}\right) ; \quad s_{\mathrm{ij}} \equiv \sin \left(\theta_{\mathrm{i}}+\theta_{\mathrm{j}}\right)
$$

\section{Solution for arm joint variables $\theta_{1}, \theta_{2}$ and $\theta_{3}$}

\subsection{Finding the approach vector of the end effector $\mathrm{A}$}

The Transformation matrix of the end effector of the robot manipulator is given as follows:

$$
{ }_{6}^{\circ} \mathrm{T}=\left[\begin{array}{cccc}
N & S & A & P \\
0 & 0 & 0 & 1
\end{array}\right]
$$

We can obtain the approach vector directly from Eqn. (8).

$$
A=\left[A_{x} A_{y} A_{z}\right]^{\mathrm{T}}
$$




\subsection{Computing the arm position vector ${ }_{a}^{0} \boldsymbol{P}$}

The position vector of the end effector is taken from Eqn. (8),

$$
{ }_{6}^{0} P=\left[P_{x} P_{y} P_{z}\right]^{\mathrm{T}}
$$

Seeing the vector relationship in Fig. 2, the vector is given as follows:

$$
{ }_{w}^{0} \boldsymbol{P}_{1}=\left|{ }_{w}^{0} \boldsymbol{P}_{1}\right| A=\left|d_{6}\right| A
$$

or

$$
\left[\begin{array}{l}
{ }_{w}^{0} \boldsymbol{P}_{1 x} \\
{ }_{w}^{0} \boldsymbol{P}_{1 y} \\
{ }_{w}^{0} \boldsymbol{P}_{1 z}
\end{array}\right]=\left[\begin{array}{l}
\left|d_{6}\right| A_{x} \\
\left|d_{6}\right| A_{y} \\
\left|d_{6}\right| A_{z}
\end{array}\right]
$$

According to Eqns. (9) and (10), the vector ${ }_{5}^{0} P$ is given as follows:

$$
{ }_{5}^{0} \boldsymbol{P}={ }_{6}^{0} \boldsymbol{P}-{ }_{w}^{0} \boldsymbol{P}=\left[\begin{array}{c}
P_{x} \\
P_{y} \\
P_{z}
\end{array}\right]-\left[\begin{array}{l}
\left|d_{6}\right| A_{x} \\
\left|d_{6}\right| A_{y} \\
\left|d_{6}\right| A_{z}
\end{array}\right]=\left[\begin{array}{l}
P_{x}-\left|d_{6}\right| A_{x} \\
P_{y}-\left|d_{6}\right| A_{y} \\
P_{z}-\left|d_{6}\right| A_{z}
\end{array}\right]
$$

Assuming that $\theta_{6}$ is equal to $90^{\circ}$, then $Z_{4}$, axis of joint 5 is always parallel to the normal vector of the end effector. We can get

$$
{ }_{w}^{0} \boldsymbol{P}_{2}=-\left|d_{5}\right| N=-\left[\begin{array}{l}
\left|d_{5}\right| N_{x} \\
\left|d_{5}\right| N_{y} \\
\left|d_{5}\right| N_{z}
\end{array}\right]
$$

From the operation of vector, we obtain

$$
{ }_{4}^{0} \boldsymbol{P}={ }_{5}^{0} \boldsymbol{P}-{ }_{w}^{0} \boldsymbol{P}_{2}
$$

Substituting Eqns. (11) and (12) for Eqn. (13),

$$
{ }_{4}^{0} \boldsymbol{P}={ }_{5}^{0} \boldsymbol{P}+\left|d_{5}\right| N=\left[\begin{array}{l}
P_{x}-\left|d_{6}\right| A_{x}+\left|d_{5}\right| N_{x} \\
P_{y}-\left|d_{6}\right| A_{y}+\left|d_{5}\right| N_{y} \\
P_{z}-\left|d_{6}\right| A_{z}+\left|d_{5}\right| N_{z}
\end{array}\right]
$$

$Z_{3}$, the axis of joint 4 , is always perpendicular to $Z_{0}$, the axis of joint 1 and $Z_{3}$ is also perpendicular to $Z_{4}$, the axis of joint 5 . Then $Z_{3}$ can be expressed the cross product of $Z_{0}$ and $Z_{4}$ as follows:

$$
Z_{3}=Z_{0} \times Z_{4}=\left[\begin{array}{c}
-N_{y} \\
-N_{x} \\
0
\end{array}\right]
$$

The vector ${ }_{w}^{0} \boldsymbol{P}_{3}$ is easily given as follows:

$$
{ }_{w}^{0} P_{3}=\left|d_{4}\right| Z_{3}=\left[\begin{array}{c}
-\left|d_{4}\right| N_{y} \\
-\left|d_{4}\right| N_{x} \\
0
\end{array}\right]
$$

Vector algebra is used to give the position vector of $\operatorname{arm}$ tip ${ }_{a}^{0} \boldsymbol{P}$ as follows:

$$
{ }_{a}^{0} \boldsymbol{P}={ }_{4}^{0} \boldsymbol{P}-{ }_{w}^{0} \boldsymbol{P}_{3}
$$


Substituting Eqns. (14) and (15) for Eqn. (16),

$$
\left[\begin{array}{c}
{ }_{a}^{0} P_{x} \\
{ }_{a}^{0} P_{y} \\
{ }_{a}^{0} P_{z}
\end{array}\right]=\left[\begin{array}{c}
P_{x}-\left|d_{6}\right| A_{x}+\left|d_{5}\right| N_{x}+\left|d_{4}\right| N_{y} \\
P_{y}-\left|d_{6}\right| A_{y}+\left|d_{5}\right| N_{y}+\left|d_{4}\right| N_{x} \\
P_{z}-\left|d_{6}\right| A_{z}+\left|d_{5}\right| N_{z}
\end{array}\right]
$$

4.3. Computing $\theta_{1}, \theta_{2}$ and $\theta_{3}$

From Eqn. (6),

$$
\underset{a}{0} \boldsymbol{P}=\left[\begin{array}{c}
c_{1}\left(a_{3} c_{23}+a_{2} c_{2}\right) \\
s_{1}\left(a_{3} c_{23}+a_{2} c_{2}\right) \\
-a_{3} s_{23}-a_{2} s_{2}+d_{1}
\end{array}\right]
$$

Equating the vector component of ${ }_{a}^{0} P$ with the components of Eqn. (17),

$$
\begin{aligned}
& { }_{a}^{0} \boldsymbol{P}_{x}=c_{1}\left(a_{3} c_{23}+a_{2} c_{2}\right) \\
& { }_{a}^{0} \boldsymbol{P}_{y}=s_{1}\left(a_{3} c_{23}+a_{2} c_{2}\right) \\
& { }_{a}^{0} \boldsymbol{P}_{z}=-a_{3} s_{23}-a_{2} s_{2}+d_{1}
\end{aligned}
$$

Dividing Eqn. (19) by Eqn. (18), we can get

$$
\frac{s_{1}}{c_{1}}=\frac{{ }_{a}^{0} P_{y}}{{ }_{a}^{0} P_{x}}
$$

Then

$$
\theta_{1}=\operatorname{atan} 2\left({ }_{a}^{0} P_{y} P_{x}\right)
$$

or

$$
\theta_{1}=\theta_{1}+180^{\circ}
$$

Squaring Eqns. (18) and (19), then adding,

$$
\begin{aligned}
\left(a_{3} c_{23}+a_{2} c_{2}\right)^{2} & ={ }_{a}^{0} \boldsymbol{P}_{x}^{2}+{ }_{a}^{0} \boldsymbol{P}_{y}^{2} \\
a_{3} c_{23}+a_{2} c_{2} & =\sqrt{{ }_{a}^{0} \boldsymbol{P}_{x}^{2}+{ }_{a}^{0} \boldsymbol{P}_{y}^{2}}
\end{aligned}
$$

To simplify the expression, let

$$
\alpha=\sqrt{{ }_{a}^{0} P_{x}^{2}+{ }_{a}^{0} P_{y}^{2}}
$$

Then,

$$
c_{23}=\frac{\alpha-a_{2} c_{2}}{a_{3}}
$$

Isolating $s_{23}$ in Eqn. (20) and employing the identity and Eqn. (22)

$$
\begin{gathered}
s_{23}^{2}+c_{23}^{2}=1 \\
s_{23}=\frac{{ }_{a}^{0} P_{z}-d_{1}+a_{2} s_{2}}{-a_{3}}
\end{gathered}
$$




$$
\begin{gathered}
\left(\frac{{ }_{a}^{0} P_{z}-d_{1}+a_{2} s_{2}}{-a_{3}}\right)^{2}+\left(\frac{\alpha-a_{2} c_{2}}{a_{3}}\right)^{2}=1 \\
\left({ }_{a}^{0} P_{z}-d_{1}\right)^{2}+2\left({ }_{a}^{0} P_{z}-d_{1}\right) a_{2} s_{2}+a_{2}^{2} s_{2}^{2}+\alpha^{2}-2 \alpha a_{2} c_{2}+a_{2}^{2} c_{2}^{2}=a_{3}^{2}
\end{gathered}
$$

Regrouping,

$$
2\left({ }_{a}^{0} P_{z}-d_{1}\right) a_{2} s_{2}-2 \alpha a_{2} c_{2}=a_{3}^{2}-a_{2}^{2}-\left({ }_{a}^{0} P_{z}-d_{1}\right)^{2}-\alpha^{2}
$$

Let

$$
{ }_{a}^{0} P_{z}-d_{1}=A, \quad \alpha=B
$$

Then,

$$
A s_{2}-B c_{2}=\frac{a_{3}^{2}-a_{2}^{2}-A^{2}-B^{2}}{2 a_{2}}
$$

Let

$$
\frac{a_{3}^{2}-a_{2}^{2}-A^{2}-B^{2}}{2 a_{2}}=-D
$$

We have now solved these types of equations and obtained the solutions as follows: (Paul (1981), Wang (1988), Wang and Lien (1987b)).

$$
\theta_{2}=\operatorname{atan} 2\left(\frac{B}{A}\right)-\operatorname{atan} 2\left(\frac{D}{ \pm \sqrt{A^{2}+B^{2}-D^{2}}}\right)
$$

To solve $\theta_{3}$, dividing Eqn. (23) by Eqn. (22), we obtain

$$
\frac{s_{23}}{c_{23}}=\frac{{ }_{a}^{0} P_{z}-d_{1}+a_{2} s_{2} /-a_{3}}{\alpha-a_{2} c_{2} / a_{3}}=\frac{{ }_{a}^{0} P_{z}-d_{1}+a_{2} s_{2}}{a_{2} c_{2}-\alpha}
$$

Then,

$$
\theta_{2}+\theta_{3}=\operatorname{atan} 2\left(\frac{{ }_{a}^{0} P_{z}-d_{1}+a_{2} s_{2}}{a_{2} c_{2}-\alpha}\right)
$$

or

$$
\theta_{3}=\operatorname{atan} 2\left(\frac{{ }_{a}^{0} P_{z}-d_{1}+a_{2} s_{2}}{a_{2} c_{2}-\alpha}\right)-\theta_{2}
$$

\section{Solution for wrist joint variables $\boldsymbol{\theta}_{\mathbf{4}}$ and $\boldsymbol{\theta}_{\mathbf{5}}$}

After solving the arm joint variables $\theta_{1}, \theta_{2}$ and $\theta_{3}$, the next step is to determine the wrist joint variables $\theta_{4}$ and $\theta_{3}$. We can get the rotation transformation matrix from Eqn. (6)

$$
{ }_{3}^{\mathbf{0}} R=\left[\begin{array}{ccc}
c_{1} c_{23} & -c_{1} s_{23} & -s_{1} \\
s_{1} c_{23} & -s_{1} s_{23} & c_{1} \\
-s_{23} & -c_{23} & 0
\end{array}\right]
$$


From Eqn. (7),

$$
{ }_{6}^{3} R=\left[\begin{array}{rcc}
-s_{4} & c_{4} c_{5} & -c_{4} s_{5} \\
c_{4} & s_{4} c_{5} & -s_{4} s_{5} \\
0 & s_{5} & c_{5}
\end{array}\right]
$$

From Eqn. (8),

$$
{ }_{6}^{0} \boldsymbol{R}=\left[\begin{array}{ccc}
N_{x} & S_{x} & A_{x} \\
N_{y} & S_{y} & A_{y} \\
N_{z} & S_{z} & A_{z}
\end{array}\right]
$$

Since the rotation matrix is orthonormal, its inverse is equal to its transpose, or

$$
\left({ }_{3}^{0} R\right)^{-1}=\left({ }_{3}^{0} R\right)^{\mathrm{T}}=\left[\begin{array}{ccc}
c_{1} c_{23} & s_{1} c_{23} & -s_{23} \\
-c_{1} s_{23} & -s_{1} s_{23} & -c_{23} \\
-s_{1} & c_{1} & 0
\end{array}\right]
$$

Then the right side of Eqn. (4),

$$
\left({ }_{3}^{0} R\right)^{-1}{ }_{6}^{0} R=\left[\begin{array}{ccc}
c_{1} c_{23} & s_{1} c_{23} & -s_{23} \\
-c_{1} s_{23} & -s_{1} S_{23} & -c_{23} \\
-s_{1} & c_{1} & 0
\end{array}\right]\left[\begin{array}{lll}
N_{x} & S_{x} & A_{x} \\
N_{y} & S_{y} & A_{y} \\
N_{z} & S_{z} & A_{z}
\end{array}\right]=\left[\begin{array}{lll}
N_{w x} & S_{w x} & A_{w x} \\
N_{w y} & S_{w y} & A_{w y} \\
N_{w z} & S_{w z} & A_{w z}
\end{array}\right]
$$

where

$$
\begin{aligned}
& N_{w x}=c_{1} c_{23} N_{x}+s_{1} c_{23} N_{y}-s_{23} N_{z} \\
& N_{w y}=-c_{1} s_{23} N_{x}-s_{1} s_{23} N_{y}-c_{23} N_{z} \\
& N_{w z}=-s_{1} N_{x}+c_{1} N_{y} \\
& S_{w x}=c_{1} c_{23} S_{x}+s_{1} c_{23} S_{y}-S_{23} S_{z} \\
& S_{w y}=-c_{1} s_{23} S_{x}-s_{1} s_{23} S_{y}-c_{23} S_{z} \\
& S_{w z}=-s_{1} S_{x}+c_{1} S_{y} \\
& A_{w x}=c_{1} c_{23} A_{x}+s_{1} c_{23} A_{y}-s_{23} A_{z} \\
& A_{w y}=-c_{1} S_{23} A_{x}-s_{1} s_{23} A_{y}-c_{23} A_{z} \\
& A_{w z}=-s_{1} A_{x}+c_{1} A_{y}
\end{aligned}
$$

According to Eqns. (27) and (30), we obtain the equation as follows:

$$
\left[\begin{array}{rcc}
-s_{4} & c_{4} c_{5} & -c_{4} s_{5} \\
c_{4} & s_{4} c_{5} & -s_{4} s_{5} \\
0 & s_{5} & c_{5}
\end{array}\right]=\left[\begin{array}{ccc}
N_{w x} & S_{w x} & A_{w x} \\
N_{w y} & S_{w y} & A_{w y} \\
N_{w z} & S_{w z} & A_{w z}
\end{array}\right]
$$

Equating elements $(1,1)$ and $(2,1)$ of Eqn. (+v),

$$
\begin{aligned}
-s_{4} & =N_{w x} \\
c_{4} & =N_{w y}
\end{aligned}
$$


Dividing Eqn. (41) by Eqn. (42) yields:

$$
\tan \theta_{4}=\frac{-N_{w x}}{N_{w y}}
$$

Then,

$$
\theta_{4}=\operatorname{atan} 2\left(\frac{-N_{w x}}{N_{w y}}\right)
$$

or

$$
\theta_{4}^{\prime}=\theta_{4}+180^{\circ}
$$

Equating elements $(3,2)$ and $(3,3)$ of eqn. $(40)$,

$$
\begin{aligned}
& s_{5}=s_{w z} \\
& c_{5}=A_{w z}
\end{aligned}
$$

Dividing Eqn. (44) by Eqn. (45),

$$
\tan \theta_{5}=\frac{S_{w z}}{A_{w z}}
$$

Then,

$$
\theta_{5}=\operatorname{atan} 2\left(\frac{S_{w z}}{A_{w z}}\right)
$$

or

$$
\theta_{5}^{\prime}=\theta_{5}+180^{\circ}
$$

\section{Discussion}

There is an exception, the singularity case in the above computation. To get the wrist position, $Z_{3}=Z_{0} \times Z_{4}$ is used. However when $Z_{0}$ is parallel to $Z_{4}, Z_{3}$ cannot be computed from this equation any more, since $N x=N y=0$ in this case, which is called robot manipulator singularity. When this case occurs, that is, $Z_{0}$ is parallel to $Z_{4}$, we may compute the wrist position ${ }_{a}^{0} P$ by using the following procedure. Figure 3 shows the projection of robot manipulator on the $X-Y$ plane. We can get

$$
\begin{aligned}
\alpha_{1} & =\tan ^{-1}\left(\frac{{ }_{4}^{0} P_{y}}{{ }_{4}^{0} P_{x}}\right), \quad \alpha_{2}=\sin \left(\frac{d_{4}}{\sqrt{{ }_{4}^{0} P_{x}^{2}+{ }_{4}^{0} P_{y}^{2}}}\right) \\
{ }_{a}^{0} P^{\prime} & =\sqrt{{ }_{4}^{0} P_{x}^{2}+{ }_{4}^{0} P_{y}^{2}-d_{4}^{2}}
\end{aligned}
$$

Then the wrist position ${ }_{a}^{0} P$ may be expressed as follows:

$$
\left[\begin{array}{l}
0 \\
a \\
{ }_{0}^{0} P_{x} \\
{ }_{a} P_{y} \\
{ }_{a}^{0} P_{z}
\end{array}\right]=\left[\begin{array}{c}
{ }_{a}^{0} P^{\prime} \sin \left(\alpha_{1}+\alpha_{2}\right) \\
{ }_{a}^{0} P^{\prime} \cos \left(\alpha_{1}+\alpha_{2}\right) \\
{ }_{4}^{0} P_{z}
\end{array}\right]
$$




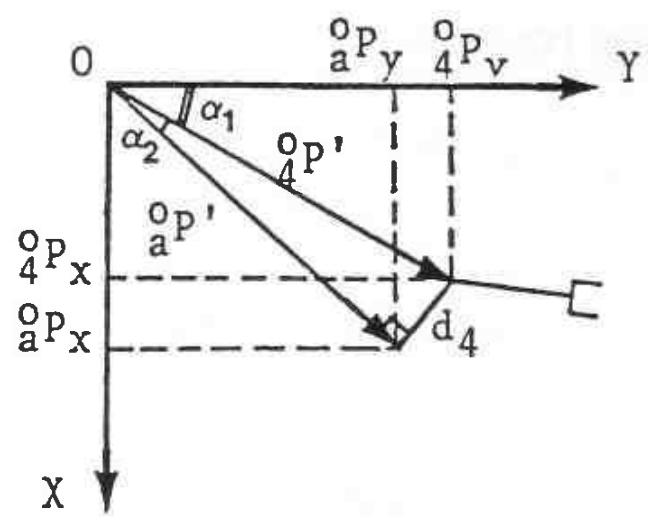

Figure 3. The projection of TR $4000 \mathrm{~S}$ on $\mathrm{X}-\mathrm{Y}$ plane.

\section{Conclusions}

A general approach to computing the inverse kinematic solutions with a closed form for robot manipulators commonly used in industry is very attractive for the robotics researchers. We have proposed such a general approach which is suitable for six-degree-of-freedom as well as five-degree-of-freedom robot manipulators with a non-spherical wrist. The general algorithm for five-degree-of-freedom robot manipulators is as follows:

Step 1. Finding the approach vector of the end effector $A$;

Step 2. Assigning zero or some suitable value to the joint variable which corresponds to the lost one degree of freedom, and then computing the arm vector ${ }_{a}^{0} P$.

Step 3. Computing the arm joint variables;

Step 4. Computing the rest of the wrist joint variables.

This approach shows that the number of computations are kept to a minimum by reducing the whole problem into a separate subproblem which in turn lowers the likelihood of error and helps to reduce the tediousness of the work.

\section{REFERENCES}

Denavit, J. and Hartenberg, R. S. (1955). A kinematic notion for lower pair Mechanisms based on matrices. J. of Applied Mechanics, Trans. ASME, 77, 215-221.

FeATHERSTONE, R. (1983). Position and velocity transformations between robot endeffector coordinates and joint angles. International Journal of Robotics Research, 2, 35-45.

Hollerbach, J. M. and SAHAR, G. (1983). Wrist-partitioned, inverse kinematic accelerations and manipulator dynamics. International Journal of Robotics Research, 2, 61-76.

Low, K. H. and DuBEY, R. N. (1986). A comparative study of generalized coordinates for solving the inverse kinematics problem of a $6 \mathrm{R}$ robot manipulator. International Journal of Robotics Research, 5, 69-88.

PAUL, R. P. (1981). Robot Manupilators: Mathematics, Programming, and Control (The MIT Press, Cambridge), pp. 40-84.

PIEPER, D. L. (1969), The Kinematics of Manipulators under Computer Control Ph.D. Dissertation, Stanford University, USA.

WANG, K. (1988). Modelling, Trajectory Planning and Computer Graphics Simulation of Robot Manipulators, Dr.ing Thesis, Norwegian Institute of Technology, Norway, ISBN 82-7119-017-2. 
WANG, K. and LIEN, T. K. (1987a). The structure Design and Kinematics of Robot manipulators. The Proceedings of International Conference on the Robotics, Dubrovnik, Yugoslavia.

WANG, K. and LiEN, T. K. (1987b). The Solution With Closed Form for Inverse Kinematics of PUMA Robot Manipulator. The Proceedings of International Conference on the Robotics, Dubrovnik, Yugoslavia. 